\title{
Physics of fully-loaded laser-plasma accelerators
}

\author{
E. Guillaume, ${ }^{1}$ A. Döpp,${ }^{1,2}$ C. Thaury, ${ }^{1}$ A. Lifschitz, ${ }^{1}$ J-P. Goddet, ${ }^{1}$ A. Tafzi, ${ }^{1}$ F. Sylla, ${ }^{3}$ \\ G. Iaquanello, ${ }^{1}$ T. Lefrou, ${ }^{1}$ P. Rousseau, ${ }^{1}$ K. Ta Phuoc, ${ }^{1}$ and V. Malka ${ }^{1}$ \\ ${ }^{1}$ Laboratoire d'Optique Appliquée, ENSTA ParisTech - CNRS UMR7639 - École Polytechnique, \\ Chemin de la Hunière, 91761 Palaiseau, France \\ ${ }^{2}$ Centro de Laseres Pulsados, Parque Cientfico, 37185 Villamayor, Salamanca, Spain \\ ${ }^{3}$ SourceLAB SAS, 86 rue de Paris, 91400 Orsay, France
}

(Received 8 December 2014; published 17 June 2015)

\begin{abstract}
While large efforts have been devoted to improving the quality of electron beams from laser plasma accelerators, often to the detriment of the charge, many applications do not require very high quality but high-charge beams. Despite this need, the acceleration of largely charged beams has been barely studied. Here we explore both experimentally and numerically the physics of highly loaded wakefield acceleration. We find that the shape of the electron spectra is strikingly independent of the laser energy, due to the emergence of a saturation effect induced by beamloading. A transition from quasi-Maxwellian spectra at high plasma densities to flatter spectra at lower densities is also found, which is shown to be produced by the wakefield driven by the electron bunch itself after the laser depletion.
\end{abstract}

DOI: 10.1103/PhysRevSTAB.18.061301

PACS numbers: 29.27.-a, 41.75.Jv, 52.38.Kd

Laser-plasma accelerators have made great progress toward higher and higher beam quality [1-4]. However, this improvement generally comes along with a reduction of charge, whereas many applications require highly charged beams. This is particularly the case in radiobiology [5], radiotherapy [6], femtosecond chemistry [7] or industrial radiography [8]. For instance, in radiography, low energy electrons can be used to produce bremsstrahlung $\mathrm{X}$-rays with energy not exceeding few $\mathrm{MeV}$ which offer large absorption contrast and do not require expansive radiation shielding. In the few $\mathrm{MeV}$ energy range, as for higher energies, the main advantages of plasma accelerators compared to conventional sources are the femtosecond duration and the micrometer source size of the electron beam. Several methods such as acceleration in cluster argon jet [9] or acceleration in pure argon or nitrogen plasmas can produce highly charged electron beams. The latter technique is based on ionization injection and trapping of electrons in a plasma created from a high-Z gas. Such an injection scheme has been proven to be a suitable way to generate electron beams with low energies and relatively high charges even for modest laser energies [10,11]. While laser wakefield acceleration in pure argon [12] or nitrogen $[13,14]$ plasma has been mainly used for generating lowcharge, quasi-monoenergetic electron beams, we point out that it is also a promising way to produce heavily-charged electron beams.

Published by the American Physical Society under the terms of the Creative Commons Attribution 3.0 License. Further distribution of this work must maintain attribution to the author(s) and the published article's title, journal citation, and DOI.
In this paper, we present an experimental and computational study of laser wakefield acceleration of highlycharged electron beams generated by ionization injection in pure argon or nitrogen. In particular the dependance of electron beam charge and energy spectrum is studied as a function of laser energy and gas density. The experimental results are reproduced by particle-in-cell (PIC) simulations, which outline two important phenomena occurring during the acceleration. First, accelerating fields saturate to a value independent from the laser energy, whereas the charge increases with the laser energy. Second, we find that for low electron density, the high charge electron beam creates its proper wakefield which changes the energy distribution.

The experiment has been performed at Laboratoire d'Optique Appliquée using the 'Salle Jaune' Ti:Sa laser system $\left(\lambda_{0}=813 \mathrm{~nm}\right.$ the laser wavelength), which delivers a linearly polarized laser pulse with a duration of $28 \mathrm{fs}$ at full width at half maximum (FWHM). The laser pulse containing $2.1 \mathrm{~J}$ is focused at the entrance of a gas jet using a $f / 10$ off-axis parabola. The $1 / e^{2}$ focal spot diameter is measured to be $22 \mu \mathrm{m}$. The energy contained in this spot diameter is $52 \%$ of the total energy. The peak laser intensity and the corresponding normalized vector potential $a_{0}$ are $8.9 \times 10^{18} \mathrm{~W} \mathrm{~cm}^{-2}$ and 2.0, respectively. Electron spectra are measured with a spectrometer consisting of a permanent magnet (760 $\mathrm{mT}$ over $20 \mathrm{~mm}$ ) combined with a phosphor screen (Kodak Lanex) imaged on a 16 bit CCD camera. The phosphor screen and detection system are calibrated so that the electron beam charge and energy distribution are measured for each shot. The gas jet is formed with a $700-\mu \mathrm{m}$-diameter sonic gas nozzle and its density profile is characterized using a Nomarski interferometer. The laser is fired at a height of $1 \mathrm{~mm}$ from the nozzle exit, leading to the formation of a $1.5 \mathrm{~mm}$ long plasma. The electron density is 
estimated considering the optical field ionization threshold for argon and nitrogen [15]. The ionization threshold for L and $\mathrm{M}$-shell electrons is below $10^{17} \mathrm{~W} \mathrm{~cm}^{-2}$, meaning that the gas can be considered preionized by the leading edge of the laser pulse up to the ionization states $\mathrm{Ar}^{8+}$ or $\mathrm{N}^{5+}$.

The laser energy is varied between $1.1 \mathrm{~J}$ and $0.2 \mathrm{~J}$ (corresponding to an intensity on target of $8.9 \times$ $10^{18} \mathrm{~W} \mathrm{~cm}^{-2}$ and $1.6 \times 10^{18} \mathrm{~W} \mathrm{~cm}^{-2}$ ) by rotating a halfwave plate followed by a polarizer in the path of the laser beam before compression. The top frame of Fig. 1 shows the evolution of the total integrated charge above $7 \mathrm{MeV}$ (lower limit of the spectrometer) obtained in an argon plasma with an electron plasma density of $1.9 \times 10^{19} \mathrm{~cm}^{-3}$ as function of the laser energy. Below $0.2 \mathrm{~J}$, the laser energy is not sufficient for the pulse to self-focus in the plasma, so the intensity is too low to trigger ionization injection. Above the threshold at $0.2 \mathrm{~J}$ we observe that the charge increases linearly by about $33 \mathrm{pC}$ per $100 \mathrm{~mJ}$ (similar results were obtained for a nitrogen plasma). However, the laser energy has low influence on the electron spectra shape. The bottom frame of Fig. 1 shows the dependency of the electron spectra shape for four different laser energies. The fact that the general shape of the spectra does not vary much is an unexpected feature, because usually the wakefield amplitude, and thus of the accelerating field, strongly depends on the laser intensity.
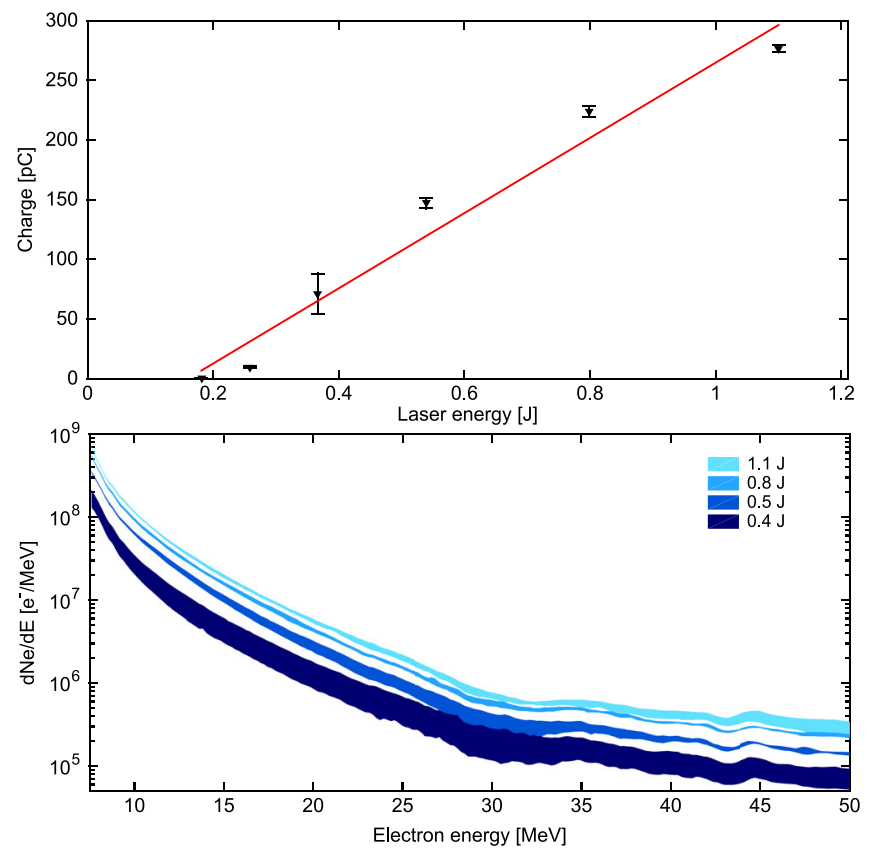

FIG. 1. Influence of the laser energy on the electron spectra for an argon plasma. (Top) Variation of the charge above $7 \mathrm{MeV}$ with the laser energy. The black triangles represent the mean charge over five consecutive shots, with the error bars showing the standard deviation. The red line is a linear fit of the charge evolution. (Bottom) Experimental spectra for four different laser energies. The colored area indicates the standard deviation over five consecutive shots.
In order to get more insight of the underlying physics of injection and acceleration in this regime, we perform simulations using the PIC code CALDERCIRC [16]. This fully electromagnetic 3D code uses cylindrical coordinates $(r, z)$ and Fourier decomposition in the poloidal direction. The simulations are performed using a mesh with $\Delta x=0.2 k_{0}^{-1}$ and $\Delta r=1.5 k_{0}^{-1}$ (with $k_{0}=1 / \lambda_{0}$ ), and two Fourier modes $(m=0-1)$. The neutral gas density profile is defined from the experimental characterization. Simulations begin with pure neutral nitrogen, which is ionized via tunnel ionization, resulting in a peak electron density of $n_{e}=1.9 \times 10^{19} \mathrm{~cm}^{-3}$. The number of macroparticles per cell before ionization is 100 , which corresponds to 500 macroelectrons per cell in the region of full ionization of the L-shell of nitrogen. The striking very weak dependency of the spectrum shape with the laser energy is also found in the simulations. The main effect of lowering the laser energy is to reduce the injected charge while the energy distribution of the electrons remains roughly constant, as well as the beam divergence. Figure 2 shows the longitudinal electric field along the laser axis toward the center of the gas jet superimposed on the electron plasma density maps, for a high energy $(1.1 \mathrm{~J})$ and a low energy $(0.4 \mathrm{~J})$ laser pulse. In the high energy case the wakefield includes around seven buckets with similar amplitude, each loaded with electrons. Note that the total charge of the

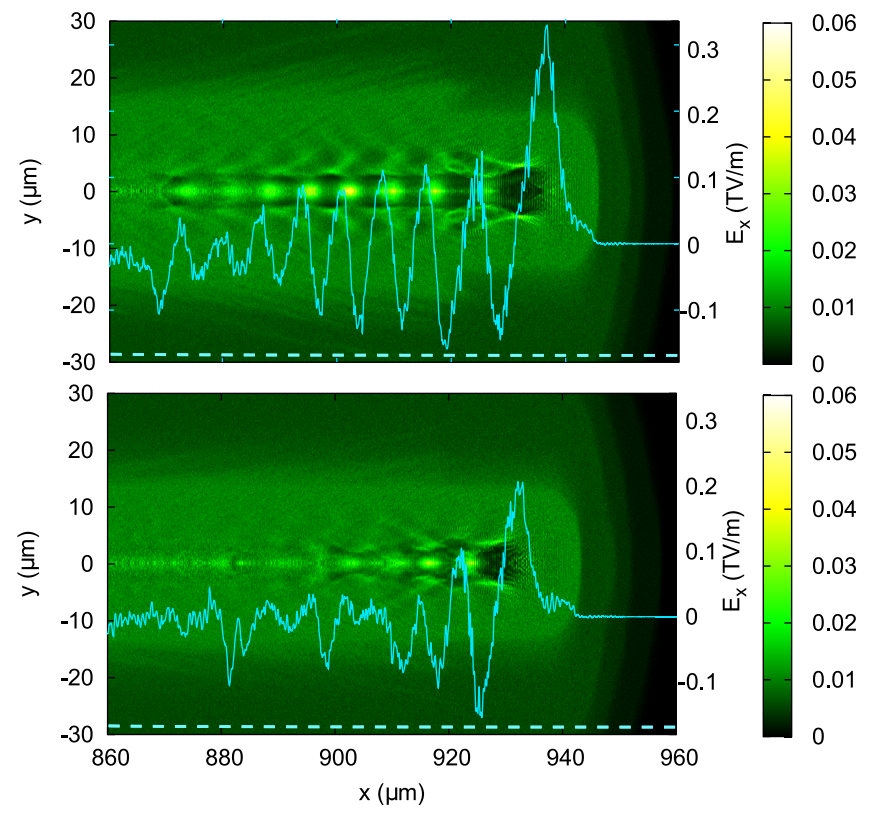

FIG. 2. Spatial distribution of electron density for laser energies of $1.1 \mathrm{~J}$ (top frame) and $0.4 \mathrm{~J}$ (bottom frame) around the middle of the gas jet (background electron density $n_{e}=1.9 \times 10^{19} \mathrm{~cm}^{-3}$ ). The superimposed plots show the longitudinal electric field on the axis (light blue). The dashed light blue line represents the theoretical injection threshold for an electron created around the middle of the gas jet $\left(E_{\text {crit }}=0.47 E_{0}\right)$. 
beam is $1 \mathrm{nC}$ in the simulation, where electrons under $7 \mathrm{MeV}$ are considered. In contrast, only two periods of the wakefield are loaded behind the low energy pulse, and the charge injected in these two buckets is roughly five times lower than in the previous case. However, as the maximum accelerating field inside the buckets is similar for both cases $\left(E_{x}=-0.17 \mathrm{TV} \mathrm{m}^{-1}\right)$, the resulting electron spectra have similar shapes. Note that although this is the major reason for the charge increase (about 3 more buckets are loaded at $1.1 \mathrm{~J}$ compare to $0.4 \mathrm{~J}$ ), the bubble size growth for larger laser energy also plays an important role: the higher the laser energy, the larger the bubble and the more electrons can be injected.

Simulations provide an explanation for this striking effect. As electrons are ionized at rest in the ionization injection mechanism [10], they must acquire sufficient energy to be injected in the wakefield. The most favorable ionization position to get injection corresponds to the point of the bubble where the accelerating field vanishes, toward the center of the bubble. Electrons resulting from ionization at that position experience the longer accelerating length. In other terms, as ionization injection is the dominant process of injection, if these electrons are not trapped, no other electron will be trapped. Because the phase velocity of the wakefield is higher than the velocity of light in the upward density gradient, the earliest ionized electrons cannot be trapped in the first plasma buckets. They slip across several buckets before being eventually injected when the wakefield slows down as the laser enters the density plateau (see Supplemental Material [17]). As more and more electrons are injected in the wakefield, the accelerating field decreases, because of electron screening. It decreases up to a point when the injection stops because electrons do not gain enough energy to be trapped in the wakefield. This saturation level is independent from the laser energy. The higher the laser energy, the more wakefield buckets can be injected, and the higher the total charge of the bunch is.

The saturation field can be estimated in the frame of the 1D nonlinear plasma wave model [18]. The minimum field for injection of an electron initially at rest at the center of the bubble is $E_{\text {crit }}=0.47 E_{0}$, where $E_{0}\left[\mathrm{~V} \mathrm{~m}^{-1}\right] \simeq$ $96 \sqrt{n_{e}\left[\mathrm{~cm}^{-3}\right]}$. For the peak density of the gas jet $\left(n_{e}=1.9 \times 10^{19} \mathrm{~cm}^{-3}\right)$, we obtain $E_{\text {crit }}=0.18 \mathrm{TV} \mathrm{m}^{-1}$, which is in agreement with the maximum accelerating field found in the simulations. As soon as the field is lower than $E_{\text {crit }}$, injection stops.

While we find that the laser energy hardly impacts the spectral distribution, we find a strong influence of the plasma density. Figure 3 shows the average electron spectra for five consecutive shots each, at $n_{e}=5.5 \times 10^{18} \mathrm{~cm}^{-3}$ (lighter blue) and $n_{e}=8.5 \times 10^{19} \mathrm{~cm}^{-3}$ (darker blue). The width of the lines corresponds to the standard deviation of the spectra. Note the very good shot-to-shot stability of these largely-charged electron beams, $50 \mathrm{pC}$ and $250 \mathrm{pC}$,

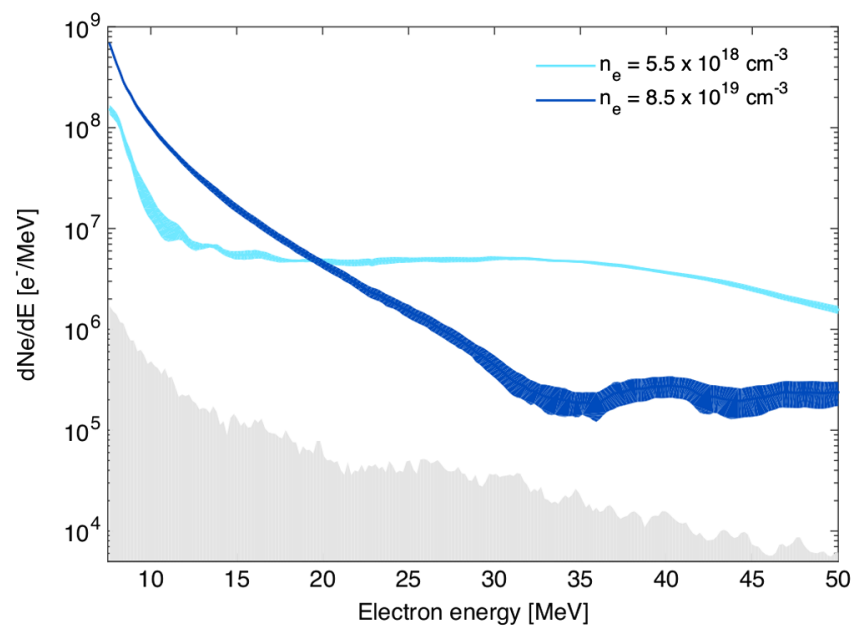

FIG. 3. Influence of the electron density on the experimental energy distribution of electrons for a nitrogen plasma at two electron densities. The colored area indicates the standard deviation over five consecutive shots. The grey shaded area indicates the detection threshold of the spectrometer.

respectively. At high electron densities, the signal decreases by roughly four orders of magnitude from $7 \mathrm{MeV}$ to $35 \mathrm{MeV}$, with the spectrum shape close to a thermal one. The quasi-Maxwellian shape of the spectrum is due to the continuous injection of electrons in multiple buckets along the acceleration. However, as we lower the electron density we enter a different regime in which the charge per $\mathrm{MeV}$ drops quickly below $12 \mathrm{MeV}$, but then is much flatter and keeps roughly the same order of magnitude up to the spectrometer cutoff. Note that the root-mean-square divergence of the beam drops from $7 \mathrm{mrad}$ to $2 \mathrm{mrad}$ at $20 \mathrm{MeV}$ when entering this regime. Again, simulations help us to reproduce the main trends of the spectrum dependency on the plasma density.

The top frame of Fig. 4 shows the variation of the electron energy distribution for two positions in the gas jet for an electron plasma density of $n_{e}=5 \times 10^{18} \mathrm{~cm}^{-3}$. In the low density case, electrons are injected around $x=600 \mu \mathrm{m}$. The ionization injection process leads to the trapping of an electron macrobunch which is composed of several distinct sub-bunches, where the separation length is approximately the local plasma wavelength $\lambda_{p}$. The multibunch structure is visible in the spatial distribution of electron density around the middle of the gas jet (middle frame of Fig. 4). When the multibunch propagates through the downward density gradient at the end of the gas jet, its structure starts to blur up. By the middle of the falling gradient of the gas jet $(x=1200 \mu \mathrm{m})$, the different subbunches form a continuous macroelectron bunch. Toward the end of the jet (see the electron density spatial distribution in the bottom frame of Fig. 4), the electron plasma density is much lower and the macrobunch, with a length close to $60 \mu \mathrm{m}$, is able to drive its own wakefield (the bunch length, of the same order than the local $\lambda_{p}$, is 

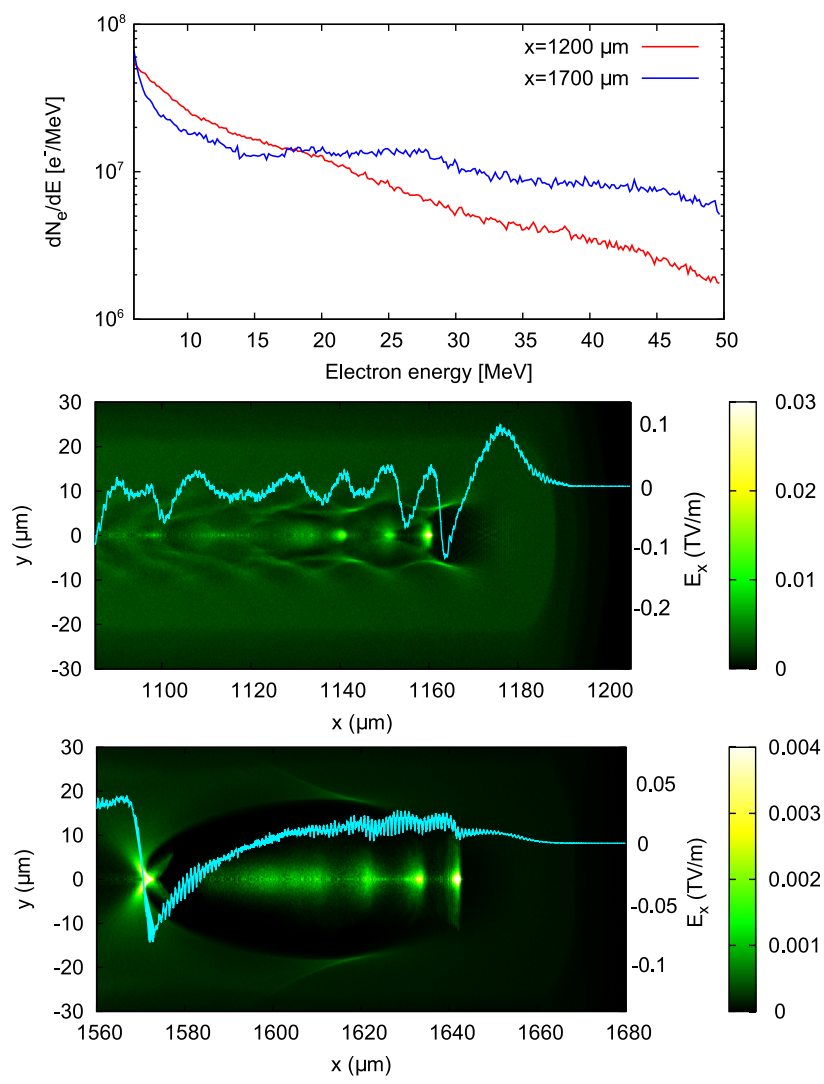

FIG. 4. (Top) Electron spectra around the center (red) and the end (blue) of the gas jet, the center of the gas jet being located at $x=900 \mu \mathrm{m}$. Spatial distribution of electron density close to the center (Middle, local electron density $n_{e}=3.5 \times 10^{18} \mathrm{~cm}^{-3}$ ) and at the end (Bottom, local electron density $n_{e}=5.2 \times 10^{17} \mathrm{~cm}^{-3}$ ) of the gas jet in the low density case. The superimposed plots show the longitudinal electric field on the axis (light blue).

resonant) [19-23]. The macrobunch, with its bunch structure still visible, can generate a very large bubble.

Due to the longitudinal field in this bubble, electrons at the tail of the macrobunch, those injected in the last buckets with the lowest energy, are accelerated. Those at the head of the bunch experience an inverse longitudinal force and are decelerated (the longitudinal electric field is positive, as seen in Fig. 4). The acceleration of low energy particles plus the deceleration of high energy ones produces this type of spectrum with a steep slope at low energy and a soft slope as high energy (as seen experimentally in Fig. 3). These effects appear in the acceleration of highly charged bunches when the electron density is low enough so that the electron wakefield can be resonantly excited. This is not the case for $n_{e}=8.5 \times 10^{19} \mathrm{~cm}^{-3}$ because the electron beam blows up before reaching the downramp density gradient (see Supplemental Material [17]). Note that for this electron density, self-focusing of the laser is not effective so the wakefield is excited over a few Rayleigh lengths, after which the laser disrupts because of ionization induced defocusing and filamentation.
In conclusion, experimental studies of the physics of heavily-charged electron bunches generated by ionization injection in pure nitrogen or argon are presented. Experiment and simulations underline two important processes occurring when these beams are accelerated. While the laser energy has direct influence on the number of cavities formed and loaded behind the pulse, the accelerating fields in each of these buckets saturate to a critical value independent from the laser energy. In consequence, by simply varying the laser energy it is possible to change the total injected charge in the beam without modifying its energy distribution. At low electron density, the electron wakefield effects are important and modify the spectrum; a multibunch electron beam with a quasi-Maxwellian spectrum transforms into a macrobunch structure containing higher energy electrons. The good shot-to-shot stability and the straightforward tunability of beam charge with the laser energy make laser wakefield acceleration in high-Z gases a promising source for various application experiments as radiobiology and radiography.

This work was supported by the European Research Council through the PARIS ERC project (Contract No. 226424), the X-Five ERC project (Contract No. 339128), LA3NET (GA-ITN-2011-289191), Areva NDE, the NANOBIODOS INCA project and by the Agence Nationale pour la Recherche through the projects ANR-10-EQPX-CILEX and FENICS ANR-12-JS040004-01.

[1] J. Faure, C. Rechatin, A. Norlin, A. Lifschitz, Y. Glinec, and V. Malka, Nature (London) 444, 737 (2006).

[2] G. R. Plateau, C. G. R. Geddes, D. B. Thorn, M. Chen, C. Benedetti, E. Esarey, A. J. Gonsalves, N. H. Matlis, K. Nakamura, C. B. Schroeder, S. Shiraishi, T. Sokollik, J. van Tilborg, C. Toth, S. Trotsenko, T. S. Kim, M. Battaglia, T. Stöhlker, and W. P. Leemans, Phys. Rev. Lett. 109, 064802 (2012).

[3] C. Rechatin, J. Faure, A. Ben-Ismail, J. Lim, R. Fitour, A. Specka, H. Videau, A. Tafzi, F. Burgy, and V. Malka, Phys. Rev. Lett. 102, 164801 (2009).

[4] A. Buck, J. Wenz, J. Xu, K. Khrennikov, K. Schmid, M. Heigoldt, J. M. Mikhailova, M. Geissler, B. Shen, F. Krausz, S. Karsch, and L. Veisz, Phys. Rev. Lett. 110, 185006 (2013).

[5] O. Rigaud, N. O. Fortunel, P. Vaigot, E. Cadio, M. T. Martin, O. Lundh, J. Faure, C. Rechatin, V. Malka, and Y. A. Gauduel, Cell Death and Disease 1, e73 (2010).

[6] O. Lundh, C. Rechatin, J. Faure, A. Ben-Ismail, J. Lim, C. De Wagter, W. De Neve, and V. Malka, Med. Phys. 39, 3501 (2012).

[7] V. Malka, J. Faure, and Y. A. Gauduel, Mutation Research/ Reviews in Mutation Research 704, 142 (2010).

[8] A. Ben-Ismaïl, O. Lundh, C. Rechatin, J. K. Lim, J. Faure, S. Corde, and V. Malka, Appl. Phys. Lett. 98, 264101 (2011). 
[9] Y. Fukuda, Y. Akahane, M. Aoyama, Y. Hayashi, T. Homma, N. Inoue, M. Kando, S. Kanazawa, H. Kiriyama, S. Kondo, H. Kotaki, S. Masuda, M. Mori, A. Yamazaki, K. Yamakawa, E. Echkina, I. Inovenkov, J. Koga, and S. Bulanov, Phys. Lett. A 363, 130 (2007).

[10] A. Pak, K. A. Marsh, S. F. Martins, W. Lu, W. B. Mori, and C. Joshi, Phys. Rev. Lett. 104, 025003 (2010).

[11] C. McGuffey, A. G. R. Thomas, W. Schumaker, T. Matsuoka, V. Chvykov, F. J. Dollar, G. Kalintchenko, V. Yanovsky, A. Maksimchuk, K. Krushelnick, V. Y. Bychenkov, I. V. Glazyrin, and A. V. Karpeev, Phys. Rev. Lett. 104, 025004 (2010).

[12] M. Mori, K. Kondo, Y. Mizuta, M. Kando, H. Kotaki, M. Nishiuchi, M. Kado, A. S. Pirozhkov, K. Ogura, H. Sugiyama, S. V. Bulanov, K. A. Tanaka, H. Nishimura, and H. Daido, Phys. Rev. ST Accel. Beams 12, 082801 (2009).

[13] H. Kotaki, Y. Hayashi, K. Kawase, M. Mori, M. Kando, T. Homma, J. K. Koga, H. Daido, and S. V. Bulanov, Plasma Phys. Controlled Fusion 53, 014009 (2011).

[14] M. Z. Mo, A. Ali, S. Fourmaux, P. Lassonde, J. C. Kieffer, and R. Fedosejevs, Appl. Phys. Lett. 100, 074101 (2012).

[15] S. Augst, D. Strickland, D. D. Meyerhofer, S. L. Chin, and J. H. Eberly, Phys. Rev. Lett. 63, 2212 (1989).

[16] A. F. Lifschitz, X. Davoine, E. Lefebvre, J. Faure, C. Rechatin, and V. Malka, J. Comput. Phys. 228, 1803 (2009).
[17] See Supplemental Material at http://link.aps.org/ supplemental/10.1103/PhysRevSTAB.18.061301 for a detailed explanation of the ionization injection process in multiple wave buckets and the simulated spatial distribution of electron density in high density plasmas.

[18] E. Esarey and M. Pilloff, Phys. Plasmas 2, 1432 (1995).

[19] S. Corde, C. Thaury, K. T. Phuoc, A. Lifschitz, G. Lambert, J. Faure, O. Lundh, E. Benveniste, A. Ben-Ismail, L. Arantchuk, A. Marciniak, A. Stordeur, P. Brijesh, A. Rousse, A. Specka, and V. Malka, Phys. Rev. Lett. 107, 215004 (2011).

[20] P. E. Masson-Laborde, M. Z. Mo, A. Ali, S. Fourmaux, P. Lassonde, J. C. Kieffer, W. Rozmus, D. Teychenn, and R. Fedosejevs, Phys. Plasmas 21, 123113 (2014).

[21] K. H. Pae, I. W. Choi, and J. Lee, Phys. Plasmas 17, 123104 (2010).

[22] I. Blumenfeld, C. E. Clayton, F.-J. Decker, M. J. Hogan, C. Huang, R. Ischebeck, R. Iverson, C. Joshi, T. Katsouleas, N. Kirby, W. Lu, K. A. Marsh, W. B. Mori, P. Muggli, E. Oz, R. H. Siemann, D. Walz, and M. Zhou, Nature (London) 445, 741 (2007).

[23] M. Litos, E. Adli, W. An, C. I. Clarke, C. E. Clayton, S. Corde, J. P. Delahaye, R. J. England, A. S. Fisher, J. Frederico, S. Gessner, S. Z. Green, M. J. Hogan, C. Joshi, W. Lu, K. A. Marsh, W. B. Mori, P. Muggli, N. VafaeiNajafabadi, D. Walz, G. White, Z. Wu, V. Yakimenko, and G. Yocky, Nature (London) 515, 92 (2014). 